\title{
Ultrassom Anorretal Tri-dimensional pode Selecionar Pacientes com Tumor no Reto após Neoadjuvância para Cirurgia de Preservação Esfincteriana?
}

\author{
Can Three-Dimensional Anorectal Ultrasound Select Patients with Rectal Tumor \\ for Sphincter-Saving Resection after Post-Chemoradiotherapy
}

\author{
STHELAMARIA MURAD-REGADAS ${ }^{1}$,FRANCISCO SÉRGIO P.REGADAS ${ }^{2}$, LUSMAR V. RODRIGUES $^{3}$, \\ FRANCISCO JEAN CRISPIN ${ }^{4}$, FRANCISCO CORACY C. MONTEIRO ${ }^{5}$, ERICO C HOLANDA ${ }^{6}$, LETIICIA OLIVEIRA ${ }^{7}$, \\ FELIPE RAMOS NOGUEIRA ${ }^{8}$
}

\begin{abstract}
${ }^{1}$ Professora Adjunto do Departamento de Cirurgia da Faculdade de Medicina da Universidade Federal do Ceara-UFC. Mestre e Doutora em Cirurgia pela UFC. Coordenadora do setor de Fisiologia anorretal da Faculdade de Medicina da UFC; ${ }^{2}$ Professor Titular do Departamento de Cirurgia da Faculdade de Medicina da Universidade Federal do CearaUFC. Coordenador da Disciplina do Aparelho Digestivo da UFC; ${ }^{3}$ Professor Adjunto do Departamento de Cirurgia da Faculdade de Medicina da Universidade Federal do Ceara-UFC. Coordenador do Serviço de Coloproctologia da Faculdade de Medicina da UFC; ${ }^{4}$ Mestre em Cirurgia pela UFC; ${ }^{5,6,7}$ Mestrando em Cirurgia pela UFC; ${ }^{8}$ Graduando em Medicina pela UFC.
\end{abstract}

\begin{abstract}
MURAD-REGADAS SM; REGADAS FSP; RODRIGUES LV; CRISPIN FJ; MONTEIRO FCC; HOLANDA EC; OLIVEIRA L; NOGUEIRA FR. Ultrassom Anorretal Tri-dimensional pode Selecionar Pacientes com Tumor no Reto após Neoadjuvância para Cirurgia de Preservação Esfincteriana? Rev bras Coloproct, 2009;29(3): 287-296.

RESUMO: Objetivo: Avaliar a resposta pós-quimioradioterapia-QT no tratamento do tumor no reto utilizando ultrassom anorretal tridimensional(US-3-D) visando definir a estratégia cirúrgica adequada. Método:Avaliou-se prospectivamente 32 pacientes com adenocarcinoma no reto médio e inferior. Realizou-se US-3-D para estadiamento e avaliação quanto à invasão no canal anal ou distância(cm) entre tumor e esfíncter anal interno-EAI: GrupoI-invasão no canal anal; GrupoII-distância menor-ou-igual 2cm, GrupoIII-distância maior 2. Foram encaminhados neoadjuvância e realizado US-3D após 50-55 dias. A escolha da estratégia cirúrgica baseou-se na resposta pós-QT e achados do US-3-D/pós-QT e comparado com histopatológico. Resultados:O US-3-D/ pós-QT coincidiu com histopatológico em 31/32, eficácia de 97\%. Evidenciou-se 26/27 casos com lesão residual, sensibilidade de 96\%, sendo 19(59\%) resposta parcial e 07 (22\%) sem resposta. Em 5/5 o US-3-D/pós-QT demonstrou resposta completa, especificidade e valor preditivo positivo $100 \%$. Valor preditivo negativo $83 \%$ pois um(3\%) caso inconclusivo. Realizou-se cirurgia de preservação esfincteriana em 16 pacientes ( 05 com resposta completa, 10 com resposta parcial e um inconclusivo) com margem maior que $2 \mathrm{~cm}$. Confirmados ao histopatológico com margem livre. $O$ índice Kappa na avaliação de linfonodos demonstrou concordância substancial(87,5\%). Conclui-se que o US-3D pode ser útil na escolha de pacientes que irão beneficiar-se com a cirurgia de preservação esfincteriana.
\end{abstract}

Descritores: Câncer retal, Ultrasom endorretal, Radioterapia, Tratamento Cirúrgico.

\section{INTRODUÇÃO}

O diagnóstico e o estadiamento completo das neoplasias de reto são indispensáveis na escolha da terapêutica. Os exames utilizados no estadiamento locoregional incluem desde o exame proctológico detalhado, ultrassonografia anorretal e/ou a Ressonância Nuclear Magnética com bobina anorretal visando selecionar pacientes para tratamento neoadjuvante (quimiorradioterapia-QT) ${ }^{(1-9)}$. Os estudos demonstram 
os benefícios da neoadjuvância, tais como: a diminuição de implantes de células tumorais durante a cirurgia, redução no tamanho da lesão possibilitando transformar tumores irressecáveis em ressecáveis, aumento nas chances de preservação esfincteriana nos pacientes com adenocarcinoma de reto inferior e redução na recidiva local ${ }^{(10-15)}$. No entanto, há dificuldades na avaliação da resposta dos tumores no reto pós-QT. O re-estadiamento quanto a invasão parietal e a identificação de linfonodos residuais utilizando exames de imagem demonstram redução na sua eficácia ${ }^{(16-26)}$. Alguns autores mencionam que as alterações produzidas na parede do reto pela ação da irradiação dificultam ou até impossibilitam a identificação das camadas da parede retal, limitando, portanto, a possibilidade de re-estadiamento das lesões ${ }^{(18,19,21)}$. Tem sido relatada eficácia entre 47.0 a $62.0 \%$ no re-estadiamento dos tumores quanto à invasão parietal pós-radioterapia utilizando ultrassom anorretal ${ }^{(16-19)}$. Gavioli e cols. ${ }^{(21)}$ utilizando transdutor linear (setorial) demonstraram que a fibrose é a base da imagem gerada no ultrassom após a radioterapia, sendo realizado, portanto o estadiamento da fibrose que pode ou não conter tumor residual. Dessa forma, relataram que o papel do US é indireto, avaliando a extensão da fibrose e por conseguinte da lesão em seu interior. Evidenciaram ainda que a visualização das camadas da parede retal sugere regressão completa da lesão. MuradRegadas e cols ${ }^{(27)}$ demonstraram em estudos preliminares o papel do ultrassom anorretal tridimensional na avaliação dos tumores de reto pós quimioradioterapia definindo padrões radiológicos na identificação de lesão residual ou resposta completa assim como as vantagens da modalidade multiplanar e aquisição automática das imagens. Portanto, esse estudo objetiva avaliar a resposta pós-QT no tratamento das neoplasia malignas no reto médio e inferior utilizando o ultrassom anorretal tridimensional visando definir a estratégia cirúrgica adequada comparando com o histopatológico.

\section{CASUÍSTICA E MÉTODO}

No período de setembro de 2005 a dezembro de 2007 , foram avaliados prospectivamente 54 pacientes com neoplasia no reto provenientes do Ambulatório de Coloproctologia do Hospital Universitário Walter Cantídio da Faculdade de Medicina da Universidade Federal do Ceará (HUWC-UFC). Destes, 32 pacien- tes (16 sexo masculino) com idade média entre 44 a 70 anos, portadores de adenocarcinoma do terço médio e distal do reto foram incluídos no estudo de acordo com protocolo previamente estabelecido pelo Serviço de Coloproctologia HUWC-UFC . Todos tiveram acesso ao Termo de Consentimento Livre e Esclarecido e aceitaram a participação na pesquisa. Realizaram ultrassom anorretal tri-dimensional (US 3-D) para estadiamento quanto ao grau de invasão parietal e comprometimento de linfonodos por um único examinador (SMMR). Após essa etapa inicial, foram encaminhados ao tratamento neoadjuvante com QT conforme protocolo previamente estabelecido. Após 50 a 55 dias do término do tratamento, foram reavaliados com novo US 3-D pelo mesmo examinador. Todos foram submetidos ao tratamento cirúrgico e a escolha da estratégia cirúrgica baseou-se na resposta pós-neoadjuvância e nos achados dos exames de US 3-D pós-QT. As peças cirúrgicas foram analisadas no Departamento de Patologia e Medicina Legal da UFC por patologista que desconhecia os resultados dos exames de US 3-D pós-QT. Os achados foram comparados e analisados.

\section{Ultrassom Anorretal Tridimensional}

Foi utilizado um equipamento de ultrassonografia Pro-Focus com transdutor com $360^{\circ}$, tipo 2050-2052, rotatório, com frequência de 9-16 MHz e distância focal variando de 2.8 a $6.2 \mathrm{~cm}$, B-K Medical (Herley, Denmark). Este transdutor realiza a aquisição da imagem de forma automática no sentido proximal-distal num segmento de $6.0 \mathrm{~cm}$, durante 50 segundos. Uma sequência de numerosas imagens paralelas transaxiais $(0,25 \mathrm{~mm})$ são adquiridas resultando numa imagem volumétrica digitalizada em forma de cubo amplamente móvel possibilitando análise em múltiplos planos, em tempo real e a revisão do exame posteriormente, tantas vezes quanto necessária, adicionando maior número de informações. Todos os pacientes foram submetidos ao enema retal duas horas antes do exame. Foram posicionados em decúbito lateral esquerdo (posição de Sims). Após toque retal, realizou-se retoscopia rígida prévia ao exame visando aspirar o resíduo fecal e/ou muco, identificar o tumor e ultrapassá-lo. Introduziu-se o transdutor por dentro do retoscopia possibilitando manter o balão insuflado na área escaneada e iniciar as aquisições das imagens. Três escaneamentos sequenciados foram adquiridos. O primeiro no segmento proximal ao tumor, visando avaliar a gordura perirretal. 
O segundo objetivando visualizar e mensurar o comprimento longitudinal do tumor e o último escaneamento para medir a distância da borda distal do tumor até a borda proximal da musculatura esfincteriana. Utilizouse o esfíncter anal interno (EAI) no quadrante posterior, para esta mensuração ${ }^{(28)}$

\section{Ultrassom anorretal tri-dimensional pré- quimiorradioterapia}

Foi avaliada o grau de invasão parietal do tumor e a identificação de linfonodos metastizados baseado no TNM preconizado por Hildebrant \& Fielf $^{(2)}$ em 1985. Avaliou-se a extensão distal quanto à presença de invasão no canal anal ou distância $(\mathrm{cm})$ entre a borda distal do tumor (qualquer quadrante) até a borda proximal do EAI no quadrante posterior. Assim, os pacientes foram distribuídos em três grupos, conforme as medições: Grupo I - invasão do canal anal; Grupo II - distância menor ou igual a 2 cm e Grupo III - distância maior que $2 \mathrm{~cm}$. Em relação aos linfonodos, estes foram considerados metastizado quando apresentavam forma arredondada, bordas irregulares e imagem com ecogenicidade semelhante à lesão primária ou hipoecóico. Ao contrário, foram considerados linfonodos inflamatórios quando apresentavam forma alongada, bordas regulares e imagem hiperecogênica central, correspondente ao hilo do linfonodo preservado.

\section{vantes}

\section{Quimioterapia e radioterapia neoadju-}

Os pacientes foram submetidos à radioterapia (40-50Gy, frações de $2 \mathrm{~Gy}$ ) pela técnica de três campos, durante um período de 06 semanas. A quimioterapia incluiu a infusão contínua de 5-fluoruacil $\left(425 \mathrm{mg} / \mathrm{m}^{2} /\right.$ day) endovenoso associado ao ácido folínico $\left(20 \mathrm{mg} / \mathrm{m}^{2} /\right.$ day $)$, aplicados durante o tratamento combinado.

\section{Ultrassom anorretal tri-dimensional pós- quimiorradioterapia}

Todos os pacientes foram submetidos novamente ao US 3-D 50 a 55 dias após o término da quimiorradioterapia. Não foi realizado o re-estadiamento ultrassonográfico (uT) baseado no TNM. Entretanto, foram avaliados quanto à resposta ao tratamento neoadjuvante, identificando regressão completa, regressão parcial, sem regressão de acordo com os padrões radiológicos previamente descrito ${ }^{(27)}$. Foi mensurado novamente a distância $(\mathrm{cm})$ entre a borda distal do tu- mor (qualquer quadrante) até a borda proximal do EAI no quadrante posterior visando selecionar pacientes que poderiam submeter-se a cirurgia com preservação esfincteriana. Avaliou-se ainda o mesorreto quanto à resposta linfonodal.

\section{Tratamento cirúrgico}

Todos os pacientes foram submetidos a tratamento cirúrgico e a escolha da técnica de preservação esfincteriana baseou-se na resposta a neoadjuvância e na distância maior do que $2 \mathrm{~cm}$ entre a borda distal do tumor (qualquer quadrante) e a borda proximal do EAI no quadrante posterior determinada no US 3D pós-QT. A peça cirúrgica foi avaliada quanto à presença de lesão residual e linfonodos ou regressão completa da lesão. Avaliou-se ainda a margem da lesão e comparouse os resultados do US 3-D pós-QT com histopatológico

\section{Análise estatística}

Calculou-se a eficácia, sensibilidade, especificidade, valor preditivo positivo e negativo comparando os achados do US 3-D pós-QT com os resultados do exame histopatológico. Valores $0 \%$ ou negativos indicam ausência de concordância e o valor máximo é $100,0 \%$. Foi utilizado o índice Kappa para avaliar o grau de confiabilidade entre o US 3-D pósQT na avaliação da presença de linfonodos positivo e o resultado anatomopatológico da peça. O valor Kappa apresenta a seguinte caracterização: < 0 não há concordância até 0,80 a 1,0 concordância quase perfeita.

\section{RESULTADOS}

\section{Ultrassom anorretal tri-dimensional pré- quimioradioterapia}

Dos 32 pacientes incluídos no estudo, o US 3D pré-quimiorradioterapia demonstrou 03 pacientes (9\%) com invasão até a camada muscular própria (uT2), $23(72 \%)$ com invasão da gordura perirretal (uT3) e 06 pacientes (19\%) com acometimento de órgãos adjacentes (uT4). Quanto à identificação de linfonodos com suspeita de metástase, evidenciou-se em 22 pacientes com imagens sugestivas de linfonodos positivos (69\%) e ausência de comprometimento de linfonodos em 10 $(31 \%)$.

Quanto à identificação de invasão no canal anal ou presença de margem entre a borda distal do tumor para borda proximal do esfíncter anal interno, identifi- 
cou-se invasão no canal anal (Grupo I) em 17 (53\%) pacientes, distância menor ou igual a $2 \mathrm{~cm}$ (grupo II) em 12 (38\%) e em 3 pacientes (9\%) o tumor localizava-se a mais de $2 \mathrm{~cm}$ do EAI (grupo III) (Figura 8).

\section{Ultrassom anorretal tri-dimensional pós- quimiorradioterapia}

O US 3-D pós-QT evidenciou regressão completa em 05 pacientes (16\%) (Figuras 1a,b, 2).Em 19 (59\%) pacientes identificou-se regressão parcial na lesão com redução maior ou igual a 10\% no comprimento longitudinal desta. Em 07(22\%) pacientes não se evidenciou resposta ao tratamento neoadjuvante (redução menor do que $10 \%$ no comprimento longitudinal da lesão) (Figuras 3a,b,c, 4a,b). Em um (3\%) paciente, o US 3-D pós-QT foi considerado inconclusivo devido a presença de espessamento parietal circunferencial e impossibilidade de distinguir inflamação de micro-lesão residual (Tabela 1). Houve concordância entre o US 3-D e o exame histopatológico em 31 pacientes $(97 \%)$.

Os resultados do US 3-D pós-QT coincidiram com os achados do histopatológico em 31/32 apresentando uma eficácia em $97 \%$ em predizer corretamente

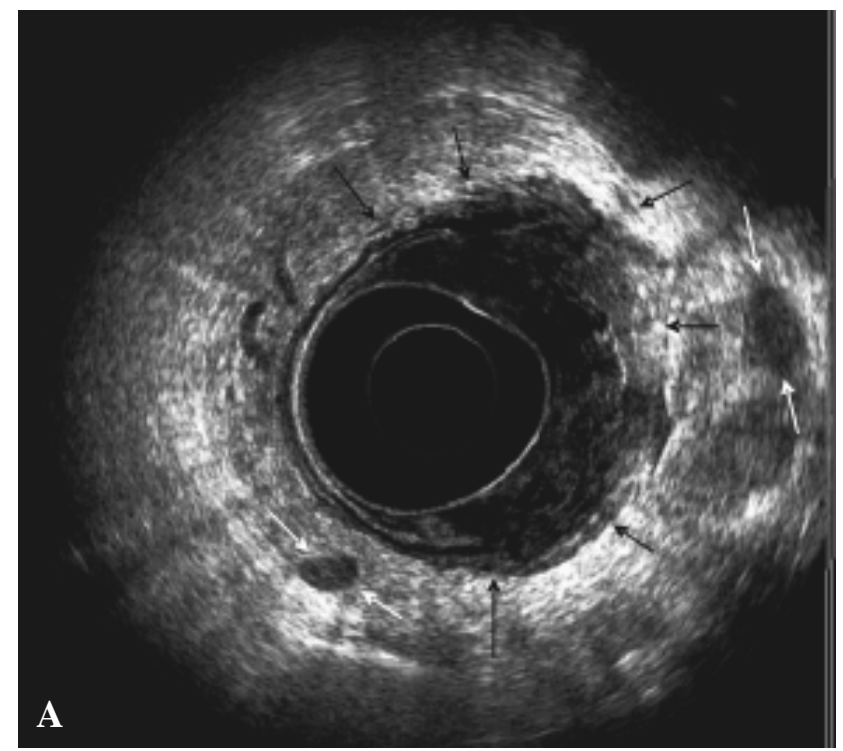

Figura 1 - Pré-quimioradioterapia - uT3 - Tumor no reto médioinferior em paciente sexo feminina, localizada no quadrante anterior e lateral direito.

a- Plano axial - Invasão na gordura perirretal (setas preta). Presença de linfonodos(2) perirretais sugestivo de metástase (setas branca). $b$ - Plano coronal e sagital - Lesão (setas) localizada $0,4 \mathrm{~cm}$ do esfíncter anal interno-EAI (d). PR- puborretal/ Tu-tumor/ EAEesfincter anal externo. a resposta tumoral ao tratamento neoadjuvante. O US 3 -D pós-quimiorradioterapia demonstrou $26 / 27$ casos com lesão residual, obtendo-se uma sensibilidade(S) de 96\%. Em 5/5 o US 3-D pós-QT evidenciou resposta completa, com especificidade-E de 100\%. Em 26 pacientes o US 3-D pós-QT demonstrou lesão residual, foi confirmado pelo histopatológico em todos, apresentando valor preditivo positivo(VPP) de 100\%. Dos 5 casos com regressão completa e um caso inconclusivo (considerado na aplicação do teste com resposta completa) demonstrado pelo US 3-D pós-QT, 5 casos foram confirmados pelo histopatológico, valor preditivo negativo de $83 \%$ (Tabela 2).

Quanto à avaliação da invasão no canal anal e mensuração na distância $(\mathrm{cm})$ entre a borda distal do tumor (qualquer quadrante) até a borda proximal do EAI no quadrante posterior pós-QT foi evidenciada mudança na distribuição dos grupos de acordo com a resposta de cada paciente (Tabela 3). Cinco pacientes $(16 \%)$ apresentaram regressão completa, sendo três que pertenciam ao Grupo I, um ao Grupo II e outro ao Grupo III. Dezenove pacientes (59\%) com regressão parcial do tumor, foi evidenciado aumento na distância da borda distal do tumor ao EAI (08 eram do Grupo I,

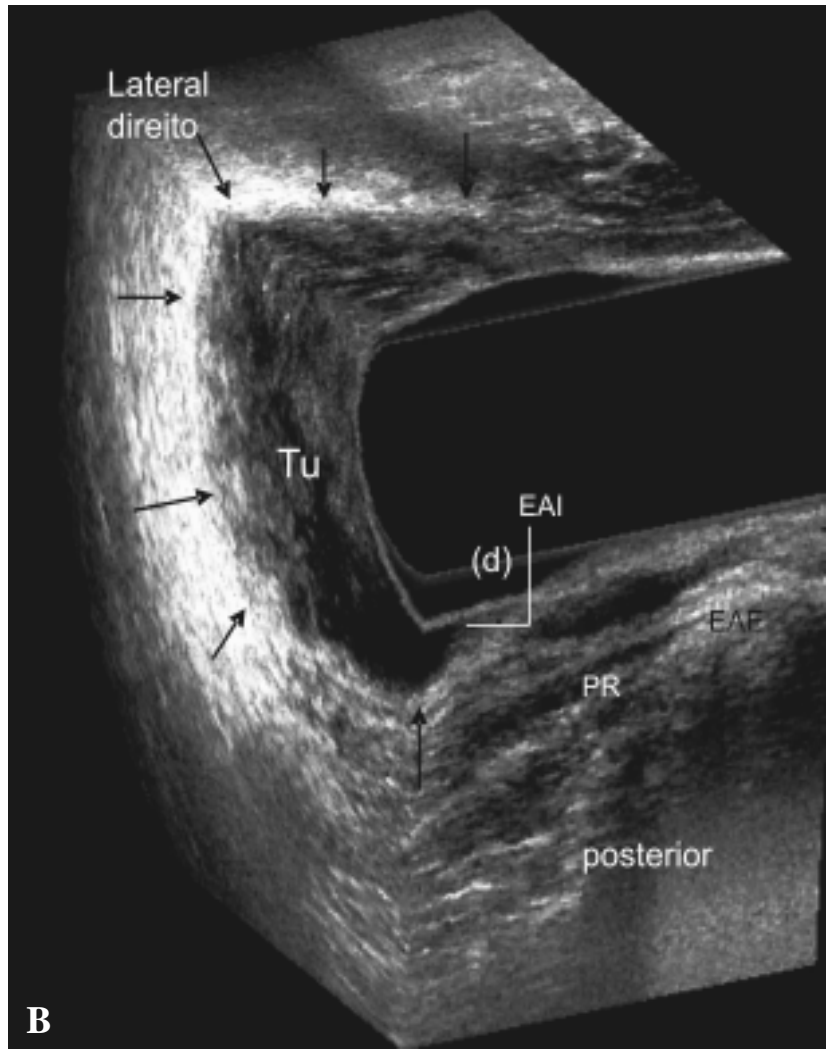




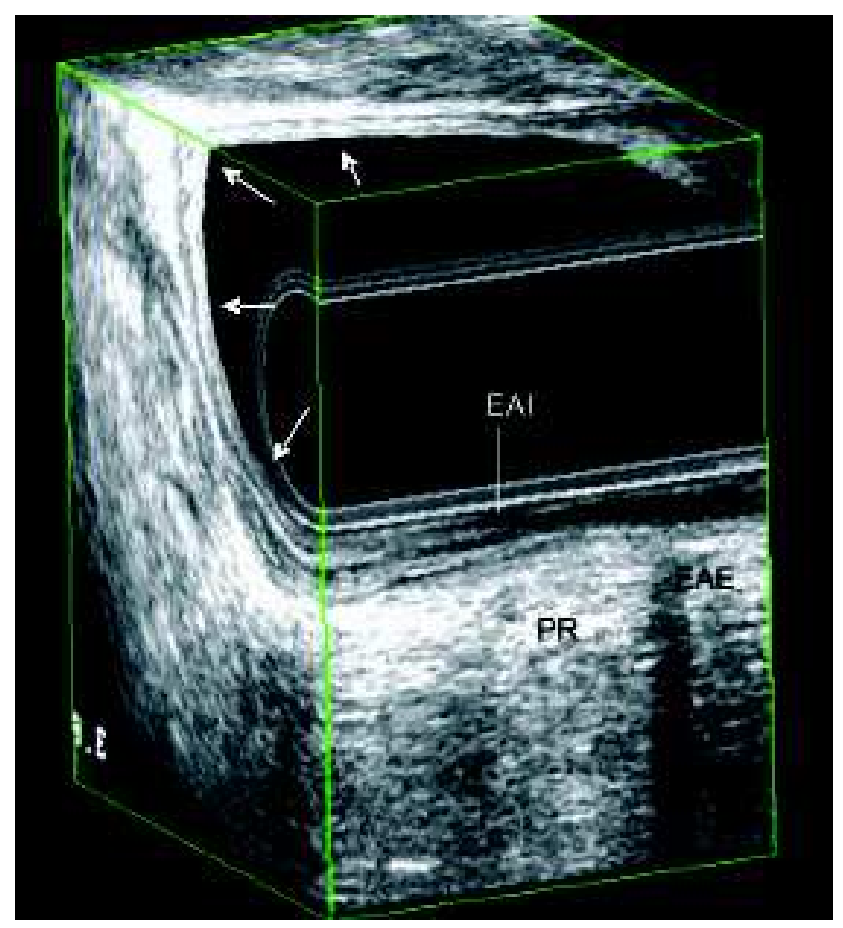

Figura 2 - Pós-quimioradioterapia - Regressão completa da lesão- visualização das camadas do reto no local prévio do tumor.

09 do Grupo II e 02 do Grupo III). Desses, 08 pacientes do Grupo II apresentaram distância do tumor ao EIA maior $2 \mathrm{~cm}$ migrando ao grupo III. Em 07 pacientes (22\%), dos quais 06 eram do Grupo I e 01 do Grupo II não apresentaram resposta ao tratamento neoadjuvante (Tabela 3 ).

Realizou-se cirurgia de preservação esfincteriana em 16 pacientes, sendo 05 com resposta completa, 10 com resposta parcial (8 eram do grupo II e 02 do grupo III) e em um paciente que, embora apresentasse imagem inconclusiva quanto à presença de tumor residual, possuía margem maior que $2 \mathrm{~cm}$ do tumor ao esfíncter (grupo III). Todos foram confirmados ao histopatológico com margem livre.

O índice de concordância Kappa na avaliação de linfonodos positivos e negativos correlacionando o US 3-D pós-QT com histopatológico demonstrou concordância substancial entre os métodos $(87,5 \%)$, com índice de 0,$692 ; 95,0 \%$ de intervalo de confiança e variação de 0,885 a 0,382 , sendo um caso falso-positivo (supra-estadiamento) e 03 falsos-negativos (subestadiamento).

\section{DISCUSSÃO}

Os exames de imagem que se propõe a reestadiar a lesão quanto a invasão parietal baseado na classificação TNM descrevem as dificuldades na interpretação das imagens devido a inflamação-fibrose, apresentando redução na sua eficácia ${ }^{(16-19)}$. Isto se deve a disposição concêntrica das camadas da parede retal, uma sobreposta a outra e a dificuldade em definir lesão tumoral e processo inflamatório de uma camada para outra. Portanto, esse estudo se propõe a reavaliar a lesão quanto identificação de lesão residual, correlacionando com a redução no tamanho da lesão e quantificando o acréscimo na margem entre a lesão

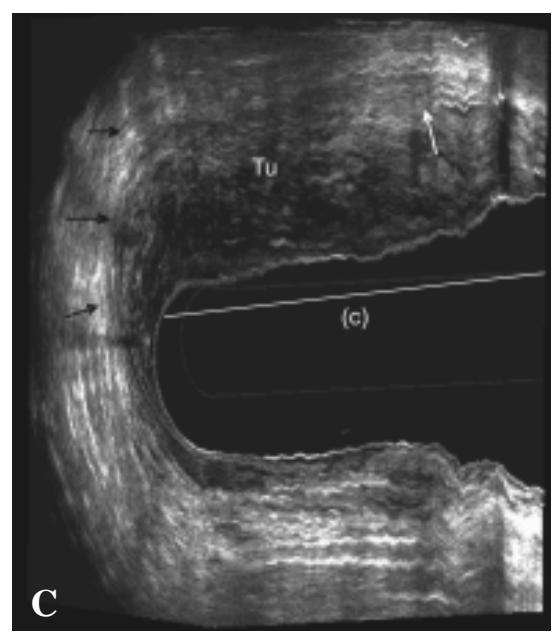

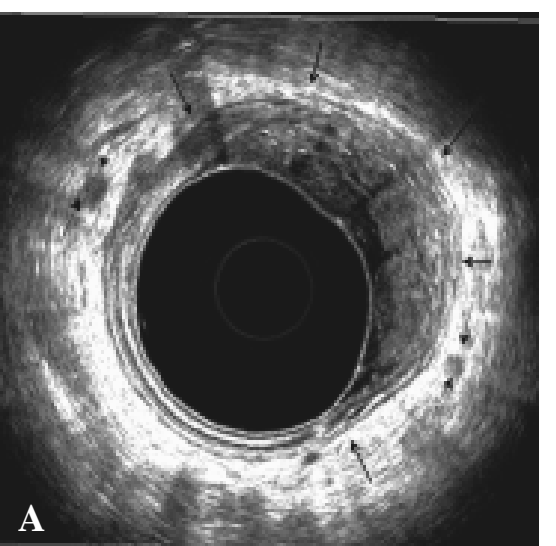

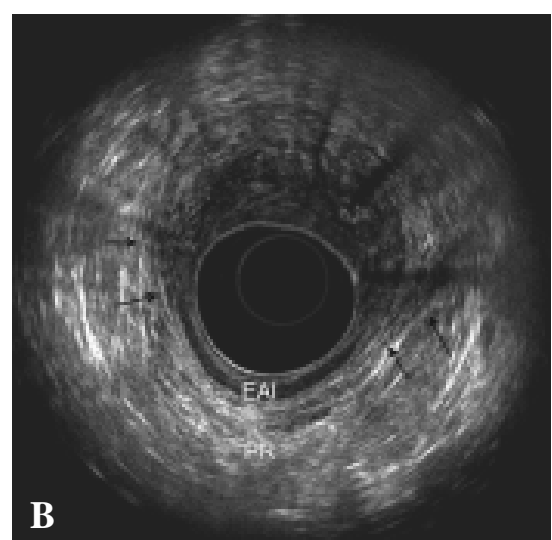

Figura 3 - Pré-quimioradioterapia - uT4N1- Tumor no reto médio-inferior no quadrante anterior e lateral esquerdo em paciente sexo feminino com invasão no canal anal (setas).

a - Plano axial - Linfonodos perirretais (setas pontilhadas).

b - Plano axial - invasão do esfíncter anal interno anterior e lateral esquerdo e invasão do puborretal lateral esquerdo. EAI-Esfíncter anal interno/PR-puborretal.

c - Plano sagital- Comprimento longitudinal da lesão (c). Invasão na vagina,perda do plano de clivagem (seta branca). 


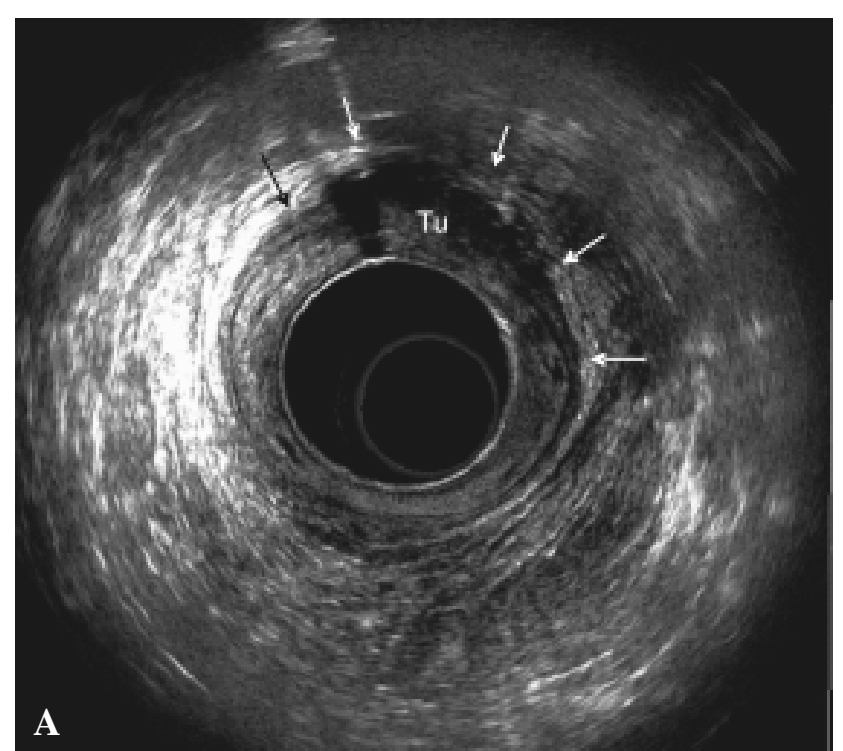

Figura 4 - Resposta parcial pós-quimioradioterapia - Lesão residual, apresentando resposta significante com redução no comprimento da lesão.

a - Plano axial.

b - Plano sagital-Apresenta distância mínima entre a borda distal do tumor e EAI posterior $(0,2 \mathrm{~cm})$ EAI- esfincter anal interno / PRpuborretal/Tu-tumor/EAE-esfincter anal externo.

residual e a musculatura esfincteriana independente do grau de invasão parietal pois não utilizou-se a classificação TNM. Foi avaliada ainda a identificação de resposta completa. Para esta avaliação utilizou-se o ultrassom anorretal tri-dimensional com $360^{\circ}$, pois apresenta frequências elevadas com até $16 \mathrm{MHz}$, distância focal com até $6,2 \mathrm{~cm}$ e aquisição automática das imagens sem a necessidade de mover o transdutor possibilitando imagens com elevada resolução espacial, estudo de múltiplos planos simultaneamente, medições exatas e a revisão do exame em tempo real.

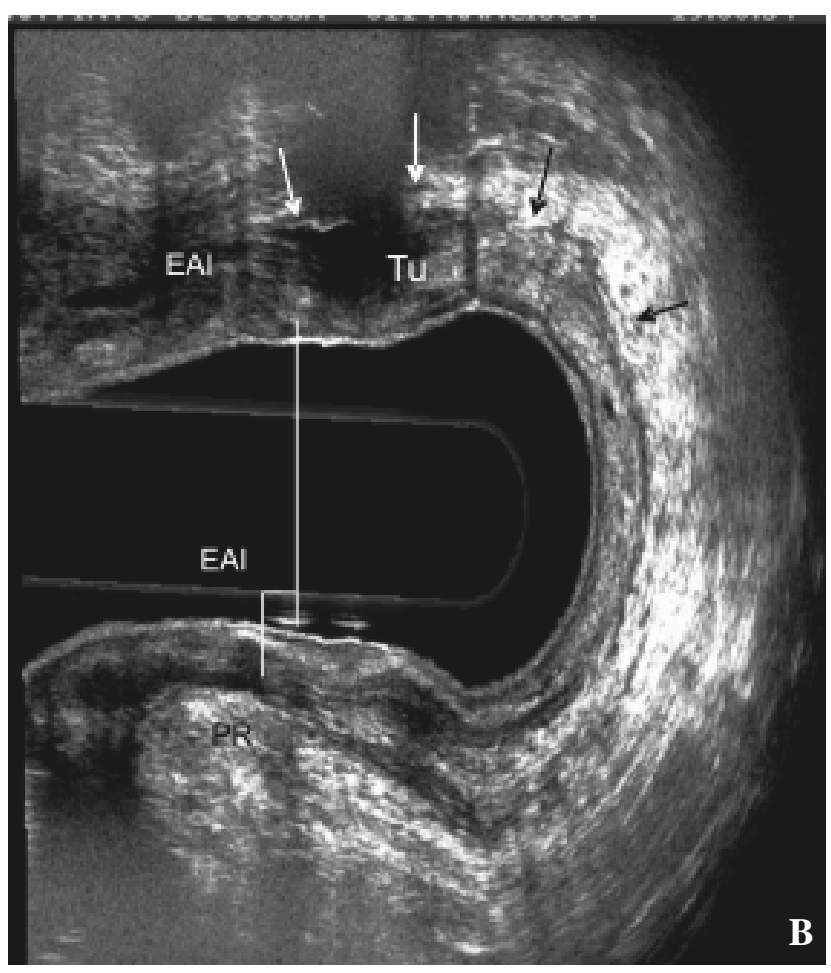

Dos 54 pacientes inicialmente selecionados para a estudo um total de 32 portadores de adenocarcinoma no reto médio e inferior com indicação de neoadjuvância concluíram completamente o protocolo previamente estabelecido. Portanto, os pacientes excluídos se deve ao abandono, interrupção ou contra-indicação do tratamento com QT; quando não realizada a avaliação pós-QT com US 3D ou a recusa na realização do tratamento cirúrgico. Todos foram conduzidos conforme a rotina do serviço de Coloproctologia HUWC-UFC. Realizou-se avaliação

Tabela 1 - Correlação entre o US 3-D pós-QT e o exame histopatológico quanto à resposta do tumor ao tratamento neoadjuvante.

\begin{tabular}{lcc}
\hline Achados & $\begin{array}{c}\text { US 3-D pós-QT } \\
\mathbf{N}^{\circ}-(\boldsymbol{\%})\end{array}$ & $\begin{array}{c}\text { Histopatológico } \\
\mathbf{N}^{\circ}-(\boldsymbol{\%})\end{array}$ \\
\hline Regressão completa & $05-(16 \%)$ & $05-(16 \%)-$ pT0N0 \\
Regressão Parcial & $19-(59 \%)$ & $19-(59 \%)-$ Lesão residual \\
Sem regressão & $07-(22 \%)$ & $07-(22 \%)-$ Lesão Residual \\
Inconclusivo & $01-(03 \%)$ & $01-(03 \%)$ - Lesão Residual \\
Total & $32-(100 \%)$ & $32-(100 \%)$ \\
\hline
\end{tabular}

US 3-D pós-QT - Ultrassom anorretal tridimensional pós-quimiorradioterapia. 
Tabela 2 - Correlação dos achados do US $3 D$ pós-QT com os resultados dos exames histopatológicos.

\begin{tabular}{lccc}
\hline & \multicolumn{2}{c}{ Exame histopatológico } & Total \\
\cline { 2 - 3 } & Presença de tumor & Ausência de tumor & \\
\hline US 3-D pós-QT & 26 & - & \\
Regressão parcial ou sem regressão & $1^{*}$ & 5 & 26 \\
US 3-D pós-QT Regressão completa & 27 & 5 & 6 \\
Total & 27 & 32 \\
\hline
\end{tabular}

* Resultado inconclusivo do US 3-D pós-QT considerado como resposta completa.

US 3-D pós-QT - Ultrassom anorretal tridimensional pós-quimiorradioterapia.

clínica, exame proctológico, US 3-D pré-neoadjuvância e colonoscopia com biópsia. Selecionou-se ao tratamento neoadjuvante aqueles apresentando estadiamento ultrassonográfico uT4, uT3 e uT2 com invasão no canal anal e/ou linfonodos positivos. Após o termino do tratamento neoadjuvante completo previamente esta- belecido foi realizado novo US 3-D após 50 a 55 dias. Os pacientes foram submetidos a ressecção cirúrgica colorretal.

Ao se correlacionar os achados do US 3-D pós-QT aos resultados do histopatológico, observou-se uma eficácia de $97 \%$ na identificação de resposta com-

Tabela 3 - Representação esquemática da correlação dos pacientes quanto a invasão no canal anal ou distância do tumor ao esfíncter anal interno pré-QT e pós-QT.

Grupo I - invasão no canal anal; Grupo II - distância igual ou menor que $2 \mathrm{~cm}$;

Grupo III - distância maior de que $2 \mathrm{~cm}$.

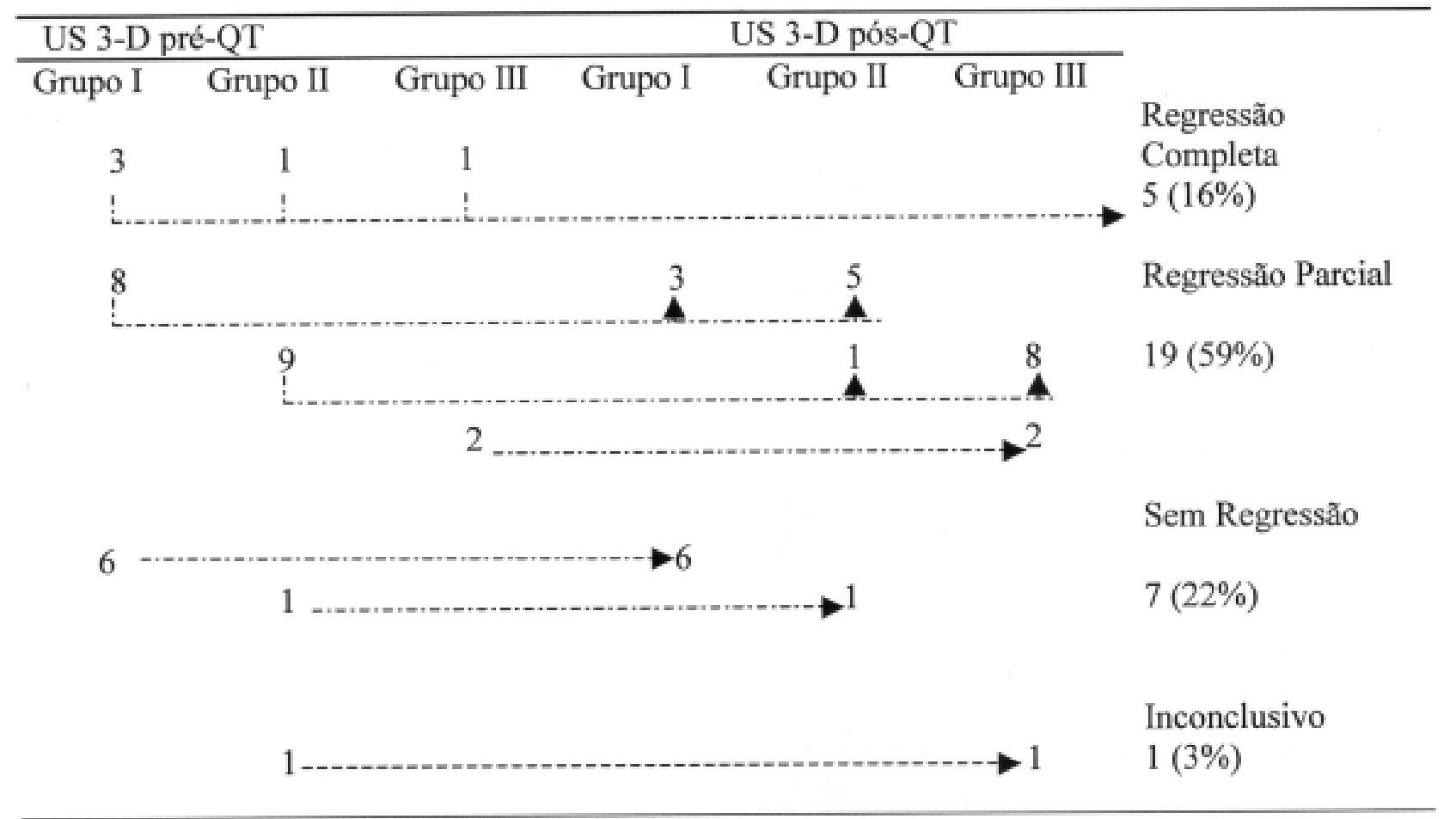

US 3-D pré-QT - Ultrassom anorretal tridimensional pré-quimiorradioterapia

US 3-D pós-QT - Ultrassom anorretal tridimensional pós-quimiorradioterapia 
pleta ou presença de lesão residual. Entre os 32 pacientes avaliados, em apenas um (3\%) o US 3-D pósQT não conseguiu excluir ou afirmar a presença de lesão residual. Este resultado ocorreu devido à presença de espessamento parietal em toda a circunferência do reto e impossibilidade de distinguir micro-lesão residual no tecido inflamatório. Considerando este achado inconclusivo como achado discordante, resultando em apenas $4 \%$ de falso negativo $(\mathrm{S}=96 \%$ ). Nos 05 casos que o exame histopatológico evidenciou ausência de lesão neoplásica, o US 3-D foi concordante em todos os casos $(\mathrm{E}=100 \%)$. Gavioli e cols ${ }^{(21)}$ demonstraram $67 \%$ de concordância com histopatológico em identificar regressão total. No entanto, Kahn e cols ${ }^{(18)}$ demonstraram apenas $17 \%$ de concordância na identificação de resposta completa na sua casuística. A confirmação de regressão completa possibilitou a cirurgia de preservação esfincteriana pois dos 5 casos, 3 desses havia invasão concomitante no canal anal. Dos 32 pacientes o US 3D pós-QT demonstrou lesão residual em 26 casos (VPP=100\%) .

Outro parâmetro avaliado pelo US 3-D foi a invasão no canal anal antes e após QT com a mensuração da distância $(\mathrm{cm})$ entre a borda distal do tumor (qualquer quadrante) até a borda proximal do EAI no quadrante posterior. Regadas e cols demonstraram a disposição assimétrica da musculatura do canal anal. Os músculos no quadrante posterior posicionam-se mais proximalmente quando comparado com anterior. Dessa forma, em qualquer que seja o quadrante que a lesão localiza-se a distância será sempre menor posteriormente e a cirurgia visa ressecção circunferencial. Apenas 07 pacientes (22\%) apresentaram regressão no comprimento longitudinal menor que $10 \%$, permanecendo 06 com invasão do canal anal (Grupo I) e 01 com distância menor que $02 \mathrm{~cm}$ do EAI (Grupo II). Nos demais 25 pacientes que apresentaram resposta ao tratamento neoadjuvante, o US 3-D evidenciou regressão no comprimento longitudinal em todos, sendo regressão completa em 05 pacientes e parcial com aumento na margem em 20 pacientes. Os limites de ressecção cirúrgica foram baseados nas imagens do US 3-D pós-QT e em todos os casos houve concordância com os laudos histopatológicos, os quais evidenciaram margens livres de doença.

Nesse tipo de avaliação, US 3-D pós-QT foi capaz de afirmar corretamente em todos os 32 pacientes (100\% dos casos) se havia invasão do canal anal ou se a distância entre o tumor e o EAI era menor, igual ou maior a $02 \mathrm{~cm}$. Mesmo no único caso inconclusivo quanto à presença ou ausência de lesão residual após QT, o US 3-D conseguiu evidenciar aumento na distância entre a imagem de espessamento parietal (inconclusiva) ao EAI resultando em cirurgia com preservação esfincteriana. Foram selecionados num total de 16 pacientes (50\%) para cirurgia com preservação esfincteriana com confirmação pelo histopatológico de margem livre de doença em todos os casos. Habr-Gama e cols ${ }^{(28)}$ tem demonstrado modificações no tratamento do câncer de reto de acordo com a resposta a neoadjuvancia apresentando resultados promissores. No entanto, no Serviço de Coloproctologia HUWC-UFC a cirurgia com ressecção colorretal ainda permanece como tratamento definitivo para tumores no reto mas a estratégia cirúrgica pode ser modificada de acordo com a resposta a neoadjuvancia. Esse estudo demonstrou avaliação quantitativa na mensuração da resposta a neoadjuvancia. Diferente das informações obtidas pelo toque retal, subjetiva, relacionadas com a experiência do examinador ou a ainda da avaliação pela retoscopia e/ou colonoscopia que visualiza a superfície da mucosa retal impossibilitando a avaliação transparietal.

A identificação de redução no estadiamento TNM, como lesão T4 regride para lesão T2 pode não modificar a estratégia cirúrgica quanto a seleção para preservação esfincteriana. No entanto, a avaliação quanto a redução no comprimento longitudinal do tumor e mensuração exata da distância entre a borda distal de lesão e a borda proximal do esfíncter anal, confirmando o acréscimo na margem podem modificar a estratégia cirúrgica e selecionar pacientes para preservação esfincteriana a partir de dados quantitativos. Não há trabalhos na literatura demonstrando essa avaliação com mensuração exata utilizando o ultra-som ou outra modalidade de exames de imagem, como a RNM.

Em relação ao estudo dos linfonodos, as imagens US 3-D pós-QT foram sugestivas de metástase linfonodal em 08 pacientes. Ao correlacionar esses resultados ao histopatológico, obteve-se uma concordância substancial de $85,7 \%$, conforme o índice de concordância Kappa. Dentre os 32 pacientes, conforme laudo histopatológico, houve 01 falso-positivo (supraestadiamento) e 03 falsos-negativos (sub-estadiamento). Os linfonodos visualizados pelo US 3-D são aqueles localizados na gordura perirretal, proximal ou distal ao tumor mas até a altura alcançada pelo transdutor. Por- 
Rev bras Coloproct Julho/Setembro, 2009
Ultrassom Anorretal Tri-dimensional pode Selecionar Pacientes com Tumor no Reto após Neoadjuvância para Cirurgia de Preservação Esfincteriana? Sthela Maria Murad-Regadas e Cols.
Vol. 29 tanto, linfonodos mesmo localizados na gordura perirretal mas posicionado proximal a posição do transdutor alcançado não foram identificados. Vale ressaltar a impossibilidade de avaliar os linfonodos da cadeia pélvica lateral, pois essa localização ultrapassa a distância focal do transdutor.

Se comparado o ultrassom anorretal tridimensional aos demais exames, ressaltam-se suas vantagens, pois é bem tolerado, aquisição de imagens rápida, de fácil realização, baixo custo e não expõe o paciente à radiação. Portanto, conclui-se que o ultrassom anorretal tridimensional adiciona importantes informações na avaliação dos tumores no reto pós-QT e pode ser útil na escolha de pacientes que irão beneficiar-se com a cirurgia de preservação esfincteriana.

\begin{abstract}
Proposal:Evaluate the post-chemoradiotherapy response for treatment of rectal tumor using three-dimensional anorectal ultrasound-3D-US to determine the best surgical approach Methods:32 patients with lower and middle rectal cancer were prospectively staged using 3D-US to identify anal canal invasion and the distance $(\mathrm{cm})$ between tumor and the internal anal sphincter-IAS, Group l:with anal canal invasion; Group II-with distance $=\mathbf{2 c m}$; Group III-with distance $>2$. They were submitted to neoadjuvant chemoradiation-CRT and the 3-D US was repeated 50-55 days later. The choice of the surgical approach was based on the post-chemoradiation response identified by the 3D-US comparing with pathologic findings. Results:The postchemoradiation/3D-US findings were concordant with pathologic results in 31/32(97\%). It was identified residual tumors in 26/ $27(96 \%$ sensibility), 19(59\%) with partial response and 7(22\%) without response. Complete response was demonstrated in 5/5 by 3D-US, with specificity and predictive valor in $100 \%$. Negative predictive valor in $83 \%$ since one $(3 \%)$ case was inconclusive. Sphincter-saving resection was performed in 16 patients, 5 with complete response, 10 with partial response and one inconclusive, with distal margin $>\mathbf{2 c m}$. The pathologic findings confirmed distal margins without tumor. It was demonstrated high concordance $(87.5 \%)$ concerning the lymph nodes evaluation(Kappa test). Conclusion: 3D-US can be useful to determine the patients who should be submitted to sphincter-saving resections.
\end{abstract}

Key words: Rectal Cancer; Endorectal Ultrasonography; Radiotherapy; Surgery Treatment.

\section{REFERÊNCIAS}

1. Rafaelsen SR, Kronborg O, Fenger C. Digital rectal examination and transrectal ultrasonography in staging of rectal câncer. A prospective blind study. Acta Radiologica 1994; 35: p 300304.

2. Hildrebant U, Fiefel G. Preoperative staging of rectal cancer by intrarectal ultrasound. Dis Colon Rectum 1985;28: p 4246 .

3. katsura Y, Yamada K, Ishizawa T, Yoshinaka H, Shimazu H. Endorectal ultrasonography for the assessment of wall invasiona nd lymphnode metastasis in rectal cancer. Dis Colon Rectum 1992; 35: p 362-368.

4. Deen KL, Madoff RD, Belmonte C, Wong WD. Preoperative staging of rectal neoplasms with endorectal ultrasonography. Seminars \& Colon Rectal Surgery 1995; 6(2): p 78-85.

5. Adams Dr, Blatchford GJ, Lin KM, Ternent CA, Thorson AG, Christensen MA. Use of preoperative ultrasound staging for treatment of rectal cancer. Dis Colon rectum 1999: 42: $\mathrm{p}$ 159-166.

6. Hunerbein M, Pegios W, Rau B, Vogl TJ, Felix R, Schlag PM. Prospective comparison of endorectal ultrasound, threedimensional endorectal ultrasound and endorectal MRI in the preoperative evaluation of rectal tumors. Preliminary results. Surg Endosc 2000; 14(11): p 1005-1009.
7. Regadas SMM, Regadas FSP, Rodrigues LV. Estadiamento ultrasonográfico de tumores de reto: Aspectos técnicos do exame e revisão da literatura. Rev bras. Coloproct 2001; 21(2): 65-9.

8. Kim JC, Cho YK, Kim SY Park SK, Lee MG. Comparative study of three-dimensional and conventional endorectal ultrasonograpy used in rectal cancer staging. Surg Endosc 2002; 16(9): p 1280-1285.

9. L. Winter ; H. Bruhn; J. Langrehr ; P. Neuhaus; R. Felix; Lopez E. Hänninen. Magnetic Resonance Imaging in Suspected Rectal Cancer: determining Tumor Localization, Stage, and Sphincter-Saving Resectability at 3-Tesla-Sustained High Resolution. Acta Radiologica 2007; 48(4): p 379 - 387.

10. Wichmann MW, Muller C, Meyer G, Straus T, Hournung H. Effect of preoperative radiochemotherapy on lymph node retrieval after resection of rectal cancer. Arch Surg. 2002; 137 : p 206-210.

11. Kaminsky-Forrett MC, Conroy T, Luporsi E, Peiffert D, Lapeyre M, Boissel P et al. Prognostic implications of downstaging following preoperative radiation therapy for operable T3-T4 rectal cancer. Int J Radiat Oncol Biol Phys.1998;42: p 935-941.

12. Bouzourene H, Bosman FT, Seelentag W, Bosman F T; Saraga E et al. Importance of tumor regression assessment in predicting the outcome in patients with locally advanced rectal carcino- 
ma who are treated with preoperative radiotherapy. Cancer. 2002;94: p 1121-1130.

13. Willett CG, Warland G, Hagan MP, Daly WJ, Coen J, Shellito PC, Compton CC. Tumor proliferation in rectal cancer following preoperative irradiation. J Clin Oncol. 1995;13: p 1417- 1424.

14. Habr-Gama A, de Souza PM, Ribeiro U Jr, Nadalin W, Gansl $\mathrm{R}$, Sousa AH Jr et al. Low rectal cancer: impact of radiation and chemotherapy on surgical treatment. Dis Colon Rectum. 1998;41: p 1087-1096.

15. Habr-Gama A, Perez RO, Kiss DE, Rawet V, Scanavini Neto A, Souza, PMSB et al. Preoperative chemoradiation therapy for low rectal cancer: impact on downstaging and sphinctersaving operations. Hepato-Gastroenterology 2004; 51: p 17031707.

16. Napoleon B, Pujol B, Berger F Valette PJ, Gerard JP, Souquet JC Accuracy of endosonography in the staging of rectal cancer treated by radiotherapy. Br J Surg 1991;78: p 785-788.

17. Williamson PR; Hellinger MD, Larach SW, Ferarra A. Endorectal ultrasound of T3 and T4 rectal cancers after preoperative chemoradiation. Dis Colon Rectum 1996;39: p 45-49.

18. Kahn H, Alexander A, Rakinic J, Nagle D, Fry R. Preoperative staging of irradiated cancers using digital rectal exam, computed tomography, endorectal ultrasound and magnetic resonance imaging does not accurately predict T0, N0 pathology. Dis Colon Rectum 1997; 40: p 140-144.

19. Rau B, Hunerbein M, Barth C, Wust P. Accuracy of endorectal ultrasound after preoperative radiochematherapy in local advanced rectal cancer. Surg Endosc 1999; 13: p 980-984.

20. Barbaro B, Schulsinger A, Valentín V, Marano P, Rotman M. The accuracy of transrectal ultrasound in predicting the pathological stage of low-lying rectl cancer after preoperative chemoradiation therapy. Int J Radiat Oncol Biol Phys 1999; 43: p1043-1047

21. Gavioli M, Bagni A, Piccagli I, Fundaro S, Natalini, G. Usefulness of endorectal ultrasound after preoperative radiotherapy in rectal cancer. Comparison between sonographic and histopathologic. Dis Colon Rectum 2000; 43: p 10751083.

22. Wheeler JMD, Warren BF, McC Morten NJ, Ekanyaka N, Kulacoglu H, Jones AC, George BD, Kettlewell MGW. Quantification of histologic regression of rectal cancer after irradiation. A propostal for a modified staging system. Dis colon Rectum 2002;45:p 1051-1056.

23. Vanagunas A, Lin DE, Stryker SJ (2004) Accuracy of endoscopio ultrasound for restaging rectal cancer following neoadjuvant chemoradiation therapy. Am J Gastroenterol 2004; 99: p 109-112.

24. Calvo FA, Domper M, Matute R, Martinez-Lazaro R, Arranz JA,. Desco M, Alvarez E, Carreras JL. 18F-FDG postitron emission tomography staging and restaging in rectal cancer treated with preoperative chemoradiation. Int J Radiat Oncol Phys 2004;58: p528-535.

25. Caprici C, Rubello D, Chierichetti F, Crepaldi G, Carpi A, Nicolini A, Mandoliti G, Polico C 2004 Restating after neoadjuvant chemoradiotherapy for rectal adenocarcinoma:role of 18-FDG PET. Biomedicine \& Pharmacotherapy 58:451457.

26. Deneck T, Rau B, Hoffmann KT, Hildebrandt B, Ruf J, Gutberlet M, Hunerbein M, Felix R, Wust P, Amthauer H. Comparison of CT, MRI and FDG-PET in response prediction of patients with locally advanced rectal cancer after multimodal preoperative therapy: is benefit in using functional imaging. Eur Radiol 2005;15: p 1658-1666.

27. Murad-Regadas, Regadas FSP, Rodrigues, LV, Barreto, RGL, Monteiro, FCC. Landim BB, Holanda, EC. Role of threedimensional anorectal ultrasonography in the ssessment of rectal cancer after neoadjuvant radiochemotherapy. Preliminary results. Surg. Endoscopy 2008; 23(6): p 1286-1291.

28. Regadas FSP, Murad-Regadas SM, Lima DMR, Silva FR, Barreto RGL, Souza MHLP, Regadas Filho FSP. Anal canal anatomy showed by three-dimensional anorectal ultrasonography Surg Endoscopy 2007;21:p 2207-2211.

29 - Habr-Gama A, Perez RO, Nadalin W, Sabbaga J, Ribeiro Jr U, Sousa Jr AHSS et al. Operative Versus Nonoperative Treatment for Stage 0 Distal Rectal Cancer Following Chemoradiation Therap Ann Surg. 2004 October; 240(4): p 711-718.

\section{Endereço para correspondência:}

STHELA MARIA MURAD-REGADAS

Rua Atilano de Moura, N-430 / Apto. 200

CEP: 60810-180

Fortaleza - CE

Tel.: (85) 3239-4372 Fone/Fax: (85) 3257-7728

E-mail: smregadas@hospitalsaocarlos.com.br 